\title{
Thirty-Six-Month Follow-up of Diaphragm Pacing with Phrenic Nerve Stimulation for Ventilator Dependence in Traumatic Tetraplegia: The Way Forward for Spinal Cord Injury Rehabilitation in a Developing Country
}

\author{
Vyom Sharma ${ }^{1}$, Haris Jafri ${ }^{2}$, Nilanjan Roy ${ }^{2}$, Manish Dangi ${ }^{3}$, Mohit Kataruka $^{1}$ \\ ${ }^{1}$ Department of Orthopaedics and Spine Surgery, Spinal Cord Injury Centre, Military Hospital Kirkee, Pune, India \\ ${ }^{2}$ Department of Surgery, Armed Forces Medical College, Pune, India \\ ${ }^{3}$ Department of Anaesthesiology and Critical Care, Indian Naval Hospital Ship Kalyani, Visakhapatnam, India
}

\begin{abstract}
Respiratory failure and chronic ventilator dependence in tetraplegics following cervical injuries located high on the spine (C1-C3) constitute significant challenges in the rehabilitation of patients given the occurrence of repeated hospitalizations and an ever-increasing financial burden. A 30-year-old man presented with posttraumatic tetraplegia following an unstable injury at the C1-C2 level with cord compression; he was managed by posterior stabilization and decompression followed by ventilator dependence and no rehabilitation until 6 months postinjury. We implanted phrenic nerve stimulator electrodes bilaterally for indirect diaphragm pacing by an implantable pulse generator that allowed for weaning from mechanical ventilation and spontaneous ventilator-free breathing at 20 weeks post-implantation and which facilitated post-tetraplegia rehabilitation. At 36 months after implantation, the patient is ventilator-free without any procedure-related complications or respiratory infections. Diaphragm pacing with phrenic nerve stimulation may be a way forward for ventilator-dependent tetraplegics in developing countries to pursue effective rehabilitation and improved quality of life.
\end{abstract}

Keywords: Phrenic nerve stimulation with diaphragm pacing; Traumatic tetraplegia; Ventilator dependence; Spinal cord injury rehabilitation

\section{Introduction}

Respiratory failure caused by high cervical (C1-C3) [1] spinal cord injury (SCI) precipitates prolonged mechanical ventilation $(\mathrm{MV})$, delaying rehabilitation $[2,3]$. The prevalence of long-term ventilation is $6 \%$ to $8 \%[4,5]$, with direct effects of prolonged MV including higher mortality and cost burden [6]. The reduced life expectancy, primarily due to pneumonia and treatment cost, also increases exponentially, further compromising the success of weaning patients from MV. An alternative method of ventilatory support in such patients involves diaphragm pacing (DP) [7]. Phrenic nerve stimulation (PNS) for diaphragmatic stimulation is one such technique by which to wean

Received May 9, 2020; Revised Sep 15, 2020; Accepted Oct 7, 2020

Corresponding author: Vyom Sharma

Department of Orthopaedics and Spine Surgery, Spinal Cord Injury Centre, Military Hospital Kirkee, Pune, India

Tel: +91-020-25818705, Fax: +91-020-26023169, E-mail: vyomsharma.479p@gov.in 

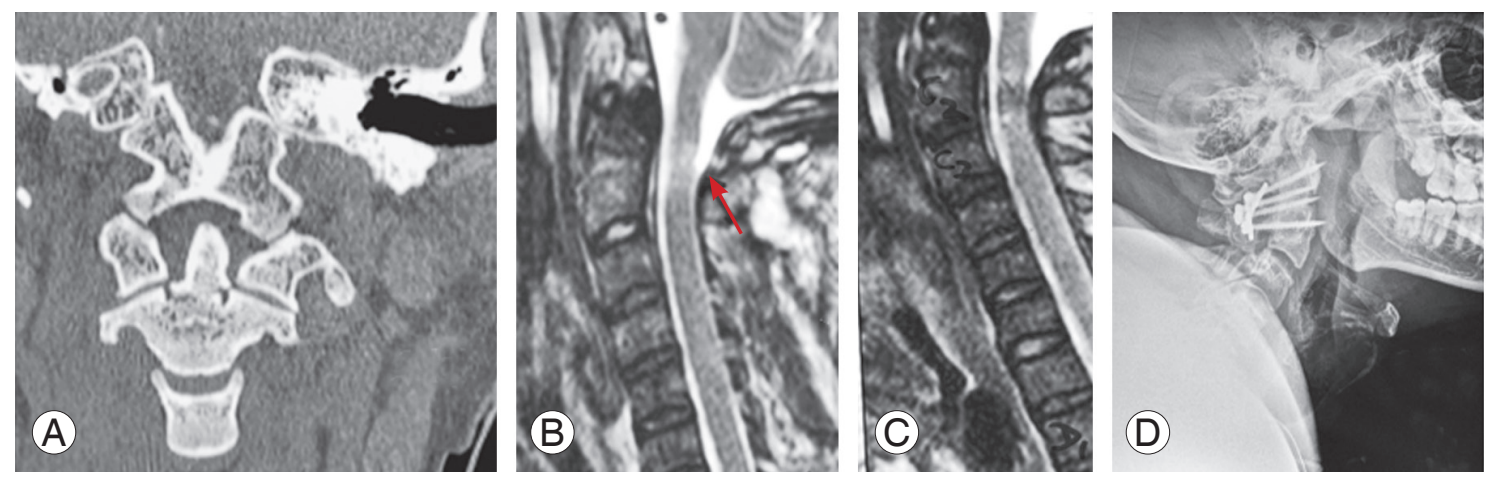

Fig. 1. (A-C) Preoperative computed tomography and magnetic resonance imaging images of $\mathrm{C} 1-\mathrm{C} 2$ fractures and cord compression (arrow). (D) Postoperative lateral radiograph of C1-C2 posterior stabilization.

tetraplegics away from chronic ventilator dependence [810]. For DP, electrode placement in the diaphragm muscle is a physiologically superior alternative to PNS, but, in the context of deploying such interventions for tetraplegics in developing countries like India, very few reports of PNS exist and those that do, lack significant long-term followup data [11-13]. We herein report our experience of DP with PNS in a ventilator-dependent patient following a high cervical SCI.

\section{Technical Note}

\section{Clinical presentation}

Our patient, a 30-year-old doctor, was brought to the emergency department of an Indian Naval Hospital in an unresponsive state following a road traffic accident, was resuscitated as per ATLS (Advanced Trauma Life Support) guidelines, and intubated before regaining spontaneous cardiac activity after 15 minutes. A detailed secondary survey revealed a Glasgow Coma Scale score of E1M1VT, sluggishly reacting pupils, flaccid extremities, absent deep reflexes, and a mute plantar reflex; the patient was subsequently diagnosed with an unstable odontoid fracture (C1-C2 instability with left C1 lamina fracture) and cord compression (Fig. 1A-C), suggesting the existence of American Spinal Cord Injury Association (ASIA) grade A tetraplegia. On the fifth day postinjury, he underwent $\mathrm{C} 1-$ C2 posterior stabilization (Goel's procedure) as shown in Fig. 1D and was continued on positive-pressure ventilation. A tracheostomy was conducted on the third postoperative day in anticipation of prolonged MV. The patient improved hemodynamically without any neurological recovery but repeatedly failed attempts to wean him from
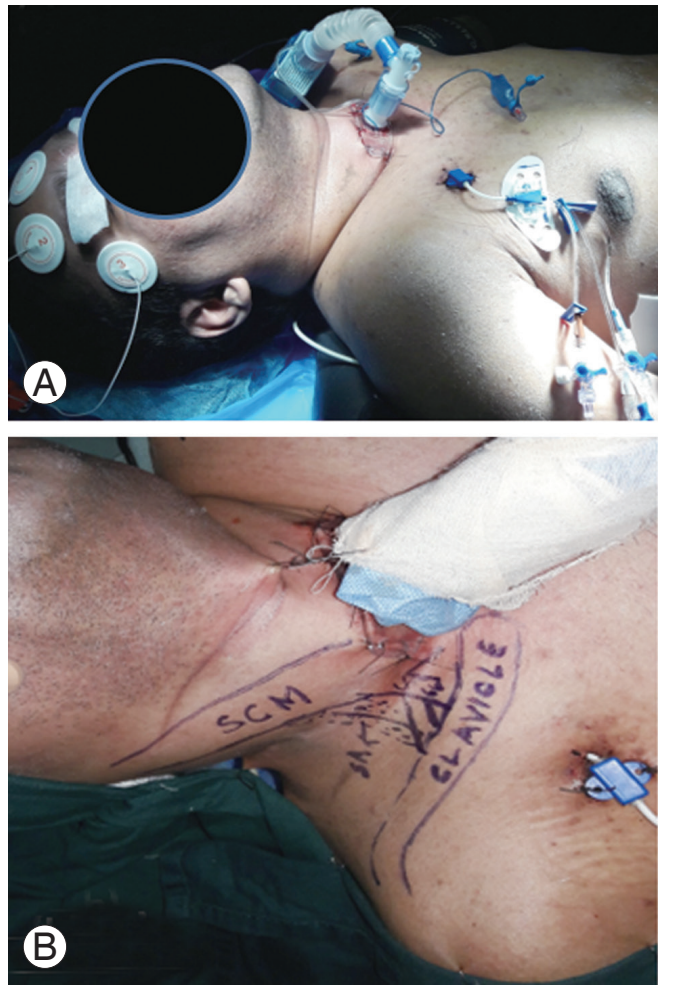

Fig. 2. (A) Supine positioning of neck in slight extension. (B) Surface marking for skin incision. Written informed consent for publication of this image was obtained from the patient.

MV. Six months later, he continued to be ventilator-dependent, tetraplegic with ASIA grade A, and bladder- and bowel-incontinent with a Spinal Cord Independence Measure (SCIM) III score of zero out of 100 points [14]. Possible modalities for weaning him from MV were explored and, with the available resources and technical expertise, DP with bilateral PNS implantation was planned. 

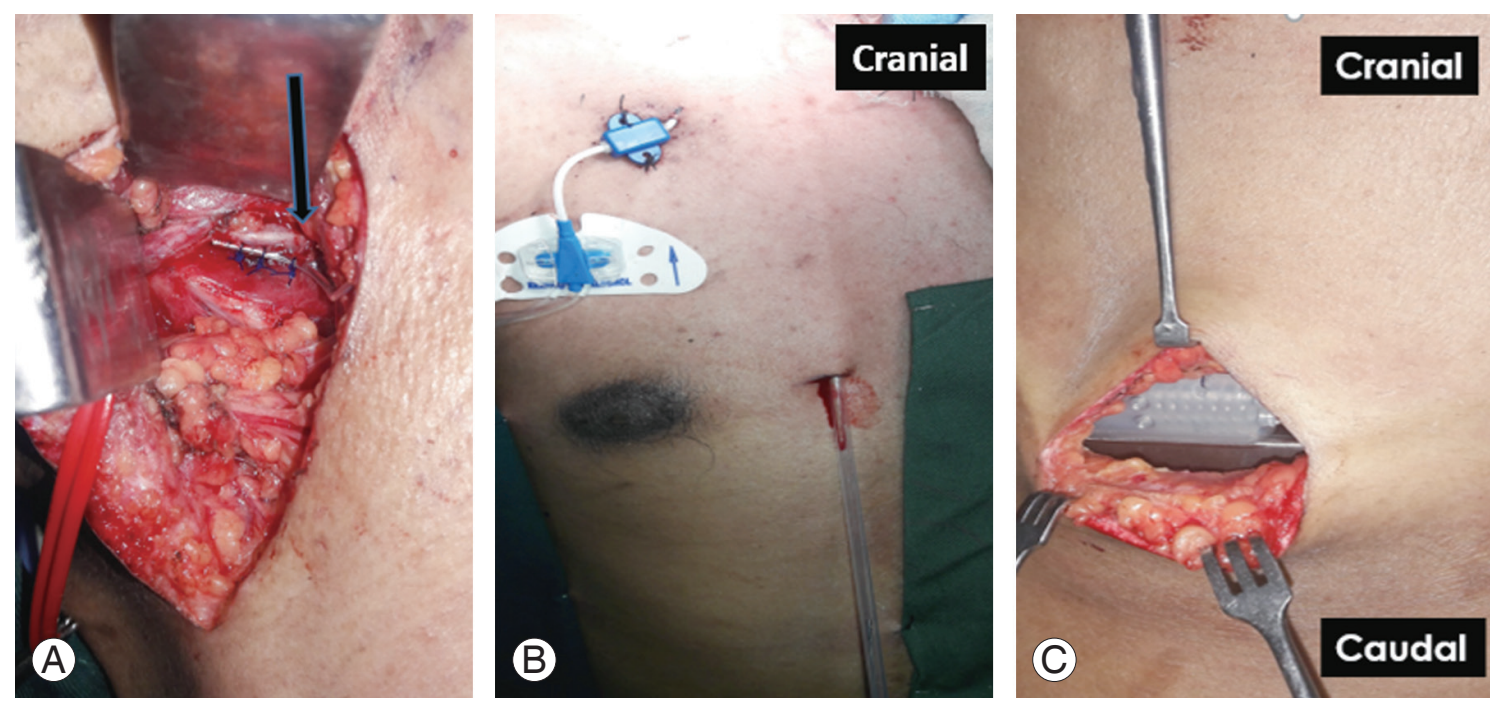

Fig. 3. (A) Placement of electrode along phrenic nerve (arrow). (B) Subcutaneous tunnel in anterior chest wall on right side. (C) Subcutaneous placement of implantable pulse generator in right hypochondrium. Written informed consent for publication of this image was obtained from the patient.

\section{Operative procedure}

The chosen procedure required an intact phrenic nerve that was identified preoperatively by ultrasonography (USG), with neck and nerve stimulation performed by a nerve stimulator under USG guidance $[15,16]$. Diaphragm jerk was identified visually and by observing diaphragm thickening on USG imaging of the lungs. Informed consent was obtained and stimulation lead implantation was performed with the patient in a supine position under general anesthesia (Fig. 2A). A skin incision was made as shown in Fig. 2B, extending from the lateral edge of the sternocleidomastoid muscle, followed by the creation of a subplatysmal flap and the identification of the C5, C6, and $\mathrm{C} 7$ spinal nerves. The phrenic nerve was then located, traversing the superior border of the scalenus medius muscle, and its integrity was assessed by monopolar electrical stimulation and the observation of contractions in the ipsilateral diaphragm. A quadripolar cylindrical electrode (Quadtrode; St. Jude Medical, Saint Paul, MN, USA) was placed along the right phrenic nerve (Fig. $3 \mathrm{~A}$ ) and tacked to the scalenus medius muscle. Successful placement of the electrode was confirmed intraoperatively by brisk downward deflection of the ipsilateral diaphragm, under fluoroscopy. A subcutaneous tunnel was created on the anterior chest wall to pass the proximal ends of the electrodes to the implantable pulse generator (IPG, EonMini Rechargeable; St. Jude Medical), as shown in Fig. 3B,

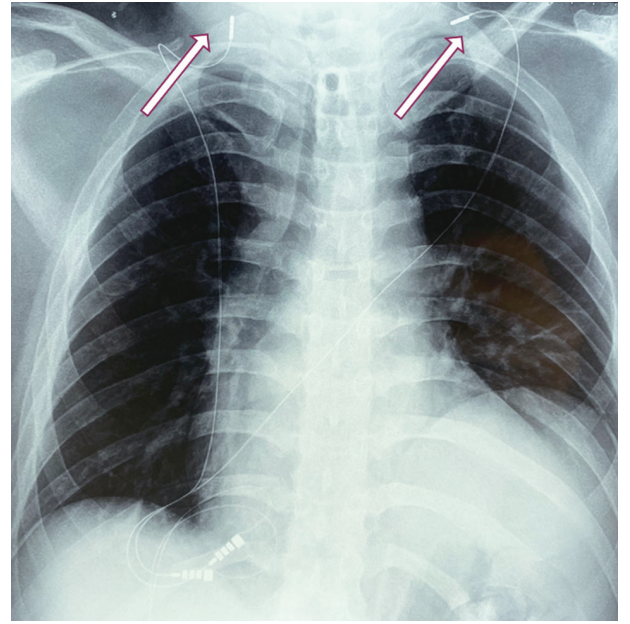

Fig. 4. Chest radiograph showing phrenic nerve stimulator electrodes connected bilaterally to implantable pulse generator in right hypochondrium (arrows).

which was placed in a subcutaneous pocket made in the right hypochondrium (Fig. 3C). A similar operation was conducted on the contralateral side to secure the correct placement of the electrodes along the left phrenic nerve and confirm their connection to the IPG in the subcutaneous pocket over the right hypochondrium (Fig. 4), confirmed on a chest radiograph.

\section{Postoperative course}

The IPG was switched on during the first postoperative 
day with an initial device setting in cyclic mode (composed of 1 second for ramp up, 2 seconds with the device on, and 3 seconds with the device off), a pulse width of 150 microseconds, a frequency of $21 \mathrm{~Hz}$, and an amplitude of $2 \mathrm{~V}$. DP was initiated as per a standardized protocol $[17,18]$ on the 10th postoperative day and diaphragm training was started (1.5-2 hr/day with an amplitude of $2.0 \mathrm{~V}$ ). The voltage was increased by $0.5 \mathrm{~V}$ each week with a corresponding increased duration of training time. The amplitude of $4.5 \mathrm{~V}$ was found to adequately contract the diaphragm to achieve adequate tidal volume and no hypercarbia after up to 8 hours of pacing.

After 6 weeks of rehabilitation, intense patient counseling, and motivation, the level of ventilator dependence was reduced to only during the night. Then, a successful trial for spontaneous ventilation during the night was conducted at 12 weeks. At this point, however, the electrode on the left side was observed to be delivering inadequate impulses, resulting in an ineffective contraction of the left hemi-diaphragm. Despite this, no respiratory muscle fatigue and an adequate blood oxygen saturation concentration at room air were noted and the patient was able to tolerate up to 96 hours of pacing before his diaphragm fatigued. The patient was ventilator-free completely at 20 weeks after implantation and a trial of spontaneous respiration without PNS and DP was attempted after patient counseling at 24 weeks after surgery; however, the patient was able to sustain a normal respiration profile only for 5 minutes at the first such attempt and in subsequent trials until the last follow-up visit. At 36 months after surgery, our patient remained reliant on DP with an SCIM III score of 7 points (4 points for respiration and 3 points for bladder management), has not required MV since, is on a capped tracheostomy tube, and is ambulant in a motorized wheelchair. During his rehabilitation, the patient's pulmonary function improved, with a change in the SCIM III respiration score from 0 to 4 points and without any pulmonary or procedure-related complications. This has enabled the patient to actively undergo a holistic rehabilitation without any episodes of anxiety or depression and with an objective improvement in the quality of life on the World Health Organization's Quality of Life-BREF (WHOQOL-BREF) questionnaire with high transformed scores domain-wise attained (i.e., 56 points in physical health, 69 points in psychological, 69 points in social relationships, and 100 points in environment) [19].

\section{Discussion}

PNS in patients requiring long-term ventilatory support due to high cervical SCI finds its basis in Caldini's work from 1786 [20] and, during the last 60 years, significant developments in the field of DP have evolved from work by Glenn et al. [21] in 1972 together with the laparoscopic implantation of electrodes directly in the diaphragm by Onders et al. [22,23]. In our patient, ventilator dependence was a major impediment in initiating post-tetraplegia rehabilitation and the option of electrode implantation directly in the diaphragm was considered to be an ideal procedure for him. However, this procedure was not feasible due to the constraints of technical expertise and the extremely limited availability of these electrodes in our country. Hence, indirect DP was considered by PNS and we improvised the use of a deep brain stimulator to act as an IPG.

An intact phrenic nerve and a healthy diaphragm are absolute prerequisites for good outcomes following this procedure but, unlike cardiac pacemakers, diaphragmatic pacemakers cannot be used immediately [5]. Instead, pacing is usually commenced at 10 days to 2 weeks after implantation and performed either unilaterally or bilaterally on alternate sides at 12-hour intervals $[17,18]$, although bilateral pacing simultaneously can also be done as seen in our patient. Usually, pacing changes are made at intervals of 10 to 14 days, depending upon the patient's tolerance level, and this conditioning phase may take anywhere from 3 to 9 months to resolve. Our patient had an extremely satisfying result regarding pacing as per a standardized protocol [17]. The anticipated challenges of such a procedure include injury to the phrenic nerve during electrode implantation, failure of the receiver or battery unit, prompt nonavailability of technical expertise to rectify the same in a developing country, and infection. Our patient experienced no such complications during followup with well-healed electrode and IPG implantation sites, a functional IPG charging unit (Fig. 5), and no episode of respiratory failure that required $\mathrm{MV}$.

Available statistics on the mortality, morbidity, and increased cost burden of ventilator-dependent SCI patients are from American or European literature $[6,24,25]$ and there is a lack of such systematic analysis from developing Asian nations. Botel et al. [26] reported an approximate daily cost per year of $\$ 357,386$ in their study on the cost of managing patients with ventilator-dependent SCI. 

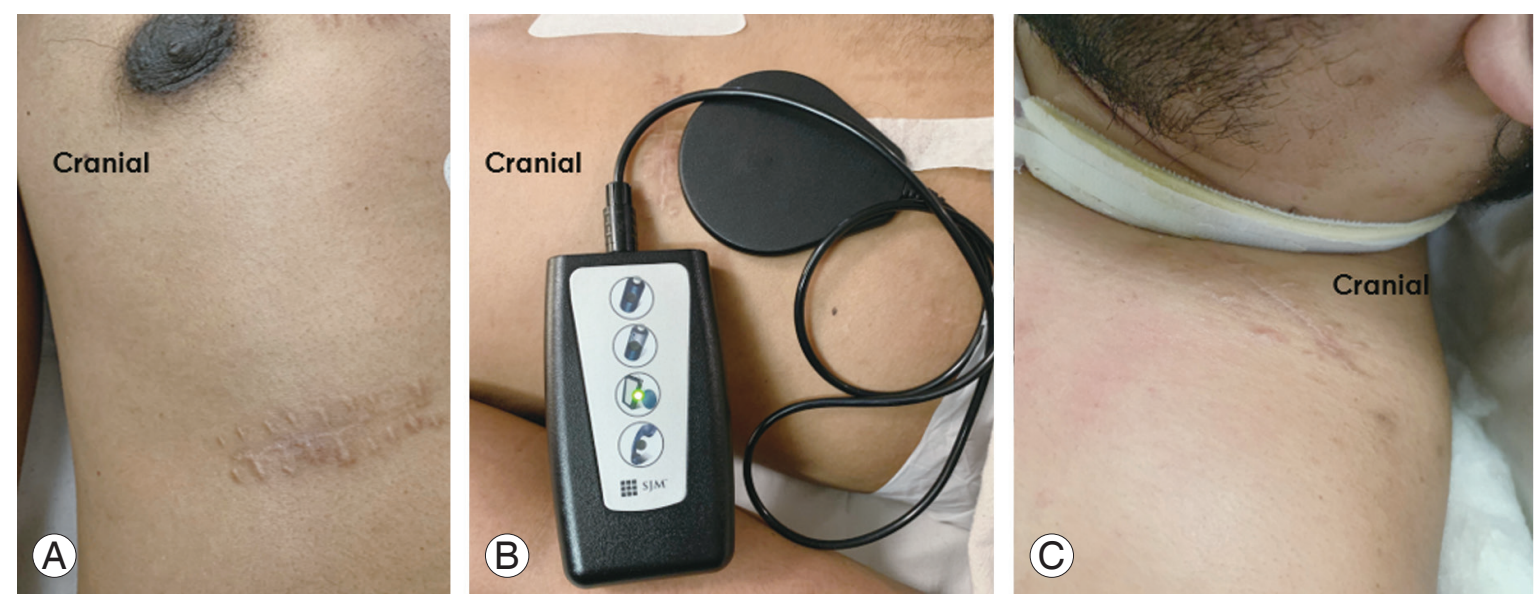

Fig. 5. (A) Well healed implantable pulse generator implantation site. (B) Functional implantable pulse generator charging unit. (C) Well healed phrenic nerve stimulation electrode implantation site. Written informed consent for publication of this image was obtained from the patient.

In our case, the initial cost of the procedure was about $\$ 13,000$ yet helped to save an annual cost of approximately $£ 10,950$ on respiratory nursing according to estimates made by Wolf et al. [27] and Similowski and Derenne [28]. The advantage of using PNS is in its inherent utility as an alternative to MV, despite the disadvantage of it being an invasive as well as an expensive procedure. However, as suggested by the numbers above, the cost of the procedure can be paid off in 1 year if the cost of MV alone is considered for the same patient [29]. In contrast with PNS, intramuscular DP is a less-invasive procedure as suggested by Onders et al. [23] but requires operators to undergo an extensive training program and learning curve in a developing country like ours where the first such procedure was only performed in 2019 by Dr. Raymond P. Onders himself [30]. In the Indian context, discharge of a ventilator-dependent tetraplegic from a hospital is a norm due to financial constraints and the lack of awareness and training concerning DP techniques. As described, we were able to avoid all these complications in our patient, with satisfactory scores attained all domains of the WHOQOLBREF questionnaire.

In conclusion, DP with PNS is a cost-effective intervention by which to facilitate ventilator-free life among tetraplegics. Early implantation of such devices may constitute a way forward for such patients but still requires surgeons to undergo training in direct diaphragm electrode pacing to facilitate faster rehabilitation and better quality of life among treated tetraplegic patients.

\section{Conflict of Interest}

No potential conflict of interest relevant to this article was reported.

\section{Acknowledgments}

This work was supported by the Department of Rehabilitation, Spinal Cord Injury Centre, Military Hospital Kirkee, Pune, India.

\section{Author Contributions}

Study concept: Vyom Sharma, Nilanjan Roy; literature search: Vyom Sharma, Haris Jafri, Manish Dangi, Mohit Kataruka; manuscript preparation: Vyom Sharma, Haris Jafri, Manish Dangi, Mohit Kataruka; manuscript editing: Vyom Sharma, Haris Jafri, Nilanjan Roy; and manuscript review: all authors.

\section{References}

1. Jackson RS, Banit DM, Rhyne AL 3rd, Darden BV 2nd. Upper cervical spine injuries. J Am Acad Orthop Surg 2002;10:271-80.

2. Epstein SK, Nevins ML. Prolonged mechanical ventilation. In: Mosenifar Z, Soo Hoo GW, editors. Practical pulmonary and critical care medicine: respiratory failure: lung biology in health and disease. New York (NY): Taylor and Francis Group; 2006. p. 187-217.

3. Jackson AB, Groomes TE. Incidence of respiratory 
complications following spinal cord injury. Arch Phys Med Rehabil 1994;75:270-5.

4. Carter RE. Experience with ventilator dependent patients. Paraplegia 1993;31:150-3.

5. Quesnel A, Veber B, Proust F, et al. What are the perspectives for ventilated tetraplegics?: a French retrospective study of 108 patients with cervical spinal cord injury. Ann Phys Rehabil Med 2015;58:74-7.

6. Berlowitz DJ, Wadsworth B, Ross J. Respiratory problems and management in people with spinal cord injury. Breathe (Sheff) 2016;12:328-40.

7. Le Pimpec-Barthes F, Legras A, Arame A, et al. Diaphragm pacing: the state of the art. J Thorac Dis 2016;8(Suppl 4):S376-86.

8. Khong P, Lazzaro A, Mobbs R. Phrenic nerve stimulation: the Australian experience. J Clin Neurosci 2010;17:205-8.

9. Le Pimpec-Barthes F, Gonzalez-Bermejo J, Hubsch JP, et al. Intrathoracic phrenic pacing: a 10-year experience in France. J Thorac Cardiovasc Surg 2011;142:378-83.

10. Romero FJ, Gambarrutta C, Garcia-Forcada A, et al. Long-term evaluation of phrenic nerve pacing for respiratory failure due to high cervical spinal cord injury. Spinal Cord 2012;50:895-8.

11. Son BC, Kim DR, Kim IS, Hong JT. Phrenic nerve stimulation for diaphragm pacing in a quadriplegic patient. J Korean Neurosurg Soc 2013;54:359-62.

12. Parthiban JK, Balasundaram S, Rajendran K, Arulselvan V, Sivakumar MN. Diaphragm pacing (phrenic nerve stimulation) in a high cervical spinal cord injury in quadriplegic patient. J Spine Surg 2017;4:1-3.

13. Bose R, Banerjee AD, Brajesh V, Narang KS, Dubey $S$, Singh VP. Phrenic nerve stimulation for diaphragmatic pacing in chronic ventilator-dependent patients. Neurol India 2018;66:1834-7.

14. Itzkovich M, Gelernter I, Biering-Sorensen F, et al. The Spinal Cord Independence Measure (SCIM) version III: reliability and validity in a multi-center international study. Disabil Rehabil 2007;29:1926-33.

15. Shaw RK, Glenn WW, Hogan JF, Phelps ML. Electrophysiological evaluation of phrenic nerve function in candidates for diaphragm pacing. J Neurosurg 1980;53:345-54.

16. Tedde ML, Vasconcelos Filho P, Hajjar LA, et al. Diaphragmatic pacing stimulation in spinal cord injury: anesthetic and perioperative management. Clinics
(Sao Paulo) 2012;67:1265-9.

17. Glenn WW, Phelps ML. Diaphragm pacing by electrical stimulation of the phrenic nerve. Neurosurgery 1985;17:974-84.

18. Taira T, Takeda N, Itoh K, Oikawa A, Hori T. Phrenic nerve stimulation for diaphragm pacing with a spinal cord stimulator: technical note. Surg Neurol 2003;59:128-32.

19. Division of Mental Health, World Health Organization. WHOQOL-BREF: introduction, administration, scoring and generic version of the assessment: field trial version, December 1996 [Internet]. Geneva: World Health Organization; 1996 [cited 2020 Sep 10]. Available from: https://apps.who.int/iris/ handle/10665/63529.

20. Schechter DC. Application of electrotherapy to noncardiac thoracic disorders. Bull N Y Acad Med 1970;46:932-51.

21. Glenn WW, Hogan JF, Loke JS, Ciesielski TE, Phelps ML, Rowedder R. Ventilatory support by pacing of the conditioned diaphragm in quadriplegia. $\mathrm{N}$ Engl J Med 1984;310:1150-5.

22. Onders RP, Khansarinia S, Weiser T, et al. Multicenter analysis of diaphragm pacing in tetraplegics with cardiac pacemakers: positive implications for ventilator weaning in intensive care units. Surgery 2010;148:893-8.

23. Onders RP, Elmo M, Kaplan C, Schilz R, Katirji B, Tinkoff G. Long-term experience with diaphragm pacing for traumatic spinal cord injury: early implantation should be considered. Surgery 2018;164:70511.

24. National Spinal Cord Injury Statistical Center. Facts and figures at a glance [Internet]. Birmingham (AL): University of Alabama at Birmingham; 2017 [cited 2020 Aug 20]. Available from: https://pdf4pro.com/ amp/view/spinal-cord-injury-facts-and-figures-at-aglance-309bc5.html.

25. Charlifue S, Apple D, Burns SP, et al. Mechanical ventilation, health, and quality of life following spinal cord injury. Arch Phys Med Rehabil 2011;92:457-63.

26. Botel U, Glaser E, Niedeggen A, Meindl R. The cost of ventilator-dependent spinal cord injuries-patients in the hospital and at home. Spinal Cord 1997;35:402.

27. Wolf C, Meiners WC, Eisenhuth J. Personal situation and situation at home of respiratory-dependent 
patients with tetraplegia: electronic versus mechanical ventilation. In: Baer GA, Exner G, editors. Functional electrical stimulation in paralysed respiratory muscles. Tampere: Tampere University Press: 2000. p. 48-58.

28. Similowski T, Derenne JP. Stimulation phrenique implantee. Med Ther 2001;7:457-69.

29. Hirschfeld S, Exner G, Luukkaala T, Baer GA. Mechanical ventilation or phrenic nerve stimulation for treatment of spinal cord injury-induced respiratory insufficiency. Spinal Cord 2008;46:738-42.
30. BioSpectrum Asia. ISIC introduces Diaphragmatic Pacing Program in India [Internet]. Pune: MM Activ Sci-Tech Communications; 2019 [cited 2020 Aug 20]. Available from: https://www.biospectrumindia. com/news/77/14306/isic-introduces-diaphragmaticpacing-program-in-india.html. 